\title{
A method of beam-couch intersection detection
}

\author{
Moorthy S. Muthuswamya) \\ Department of Radiation Oncology and Pittsburgh Cancer Institute, University of Pittsburgh, Pittsburgh, \\ Pennslyvania 15213 and Department of Radiation Oncology, University of Michigan, Ann Arbor, \\ Michigan 48109
}

Kwok L. Lam

Department of Radiation Oncology, University of Michigan, Ann Arbor, Michigan 48109

(Received 28 July 1997; accepted for publication 12 November 1998)

\begin{abstract}
At the time of treatment planning it would be useful to know whether part of the treatment beam passes through the patient/couch support assembly before it passes through the patient. In the previous work of Yorke, the range of gantry angles leading to beam-couch intersection was found as a function of couch translation for symmetric field sizes and for zero couch rotation. Yorke's method has been extended to include couch rotation, dual independent jaws, and multi-leaf collimator (MLC) field shapes. In addition, the new method is also applicable in the situation of the couch top located above the isocenter. For a clinically treatable, $20 \times 20 \mathrm{~cm}$ field configuration in a linac, the range of gantry angles leading to beam-couch intersection are different by 6.7 degrees for a couch rotation angle of 25 degrees when compared to no couch rotation. The new method agrees with data within the setup and measurement uncertainties for a variety of field sizes including an oval shaped MLC field, and various couch locations, couch, and collimator rotation angles.
\end{abstract} (C) 1999 American Association of Physicists in Medicine. [S0094-2405(99)00202-3]

Key words: beam-couch intersection, treatment planning

\section{INTRODUCTION}

With the advent of three-dimensional (3D) treatment planning, there is an increased use of oblique/nonaxial fields requiring couch rotation. Out of the many possible treatable and nontreatable couch-gantry locations, a generalized collision avoidance algorithm by Humm $^{1}$ can be used to choose diverse couch-gantry angle combinations that are treatable. Also, a graphical simulation approach toward collision avoidance has been developed. ${ }^{2,3}$ However, for the treatable configurations, there still is a possibility that part of the beam could go through the patient support assembly (PSA) before entering the patient. This leads to attenuation and scattering of the therapy beam resulting in a different dose distribution (including a larger superficial dose) than intended.

The Varian Clinac 2100 C/D "tennis racket" part of the treatment couch has a pair of supporting rails located under the couch edges. There are also steel rails placed along the sides of the couch for mounting supporting devices. All of these can attenuate the beam. In the work of Yorke, ${ }^{4}$ analytical expressions for the range of gantry angles leading to beam-PSA intersection were found as a function of transverse and vertical couch translation for symmetric field sizes and zero couch rotation. In this work, Yorke's model has been extended to include couch rotation, dual independent jaws, and MLC field shapes. The current method has been tested for the specific geometry of a Varian Clinac 2100 C/D. A FORTRAN program incorporating the analytical expressions was developed and tested. In the previous work ${ }^{4}$ couch configurations with the couch located only below the isocenter were considered. The algebraic approach outlined here is ap- plicable for situations of couch located above the isocenter as well.

Not under consideration here is part of the field lying outside the longitudinal boundaries of the tennis racket. The length of the tennis racket in a Varian Clinac $2100 \mathrm{C} / \mathrm{D}$ machine is $62 \mathrm{~cm}$, adequate enough to cover the largest diagonal field of $56 \mathrm{~cm}(40 \times 40 \mathrm{~cm}$ field $)$. So, the patient could be moved along the longitudinal direction of the couch so that the field does not extend beyond the longitudinal boundaries of the tennis racket.

\section{ANALYTICAL METHOD}

The coordinate systems defined by Siddon ${ }^{5}$ are used here. The origins of all coordinate systems are located at the isocenter. The room/fixed coordinate system is shown in Fig. 1. There are couch, gantry, and collimator coordinate systems that rotate with the couch, the gantry, and the collimator, respectively. The subscript $r, g$, and $c$ associated with various variables denote room, gantry, and collimator coordinate systems, respectively. All coordinate systems coincide with each other when the gantry is at 0 degrees (x-ray beam irradiating vertically downwards), the collimator at 0 degrees, and the couch at 0 degrees (when the longest dimension of the couch is parallel to the $y$ axis in the room coordinate system). Clockwise rotations of the couch, the gantry, and the collimator are positive when viewing from the isocenter. For the collimator angle of 0 degrees, the direction of motion of the $X$ and $Y$ jaws of the collimator are parallel to the $x$ and $y$ axes of the room coordinate system.

In Figs. 2(a) and 2(b), the PSA consists of two steel bars and the frame of the tennis racket. The beam ray and other 


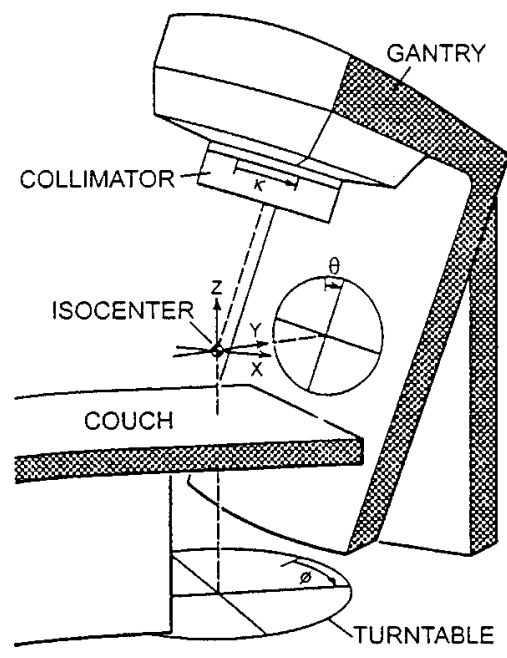

FIG. 1. The gantry-couch system of a therapy machine viewed in the room coordinate system located at the isocenter. [This figure was processed and presented with permission from Siddon (Ref. 5).]

information displayed in these figures are the projections in the $x z$ plane at the isocenter. The required machine dependent parameters of the couch and couch support assembly are: Tennis racket bar support half-width $\left(w_{b}\right.$, half the lateral distance between the corners $A$ and $C)$, its height $\left(h_{b}\right.$,

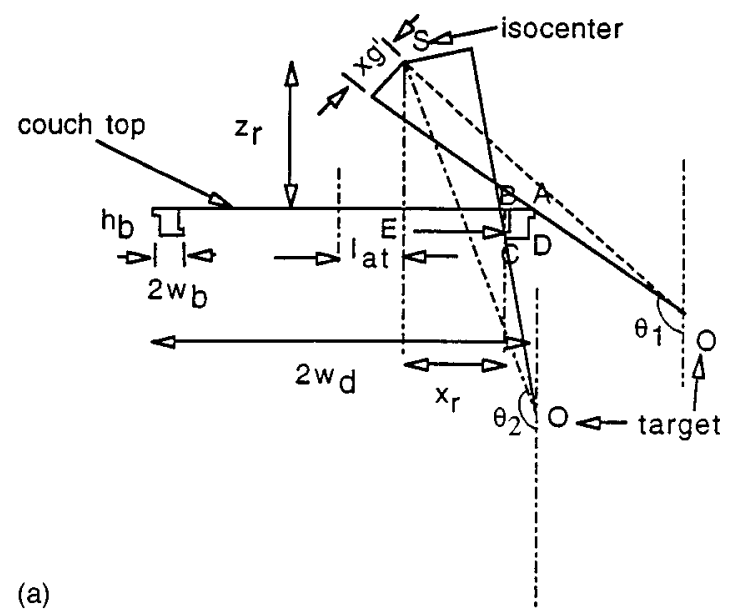

(b)

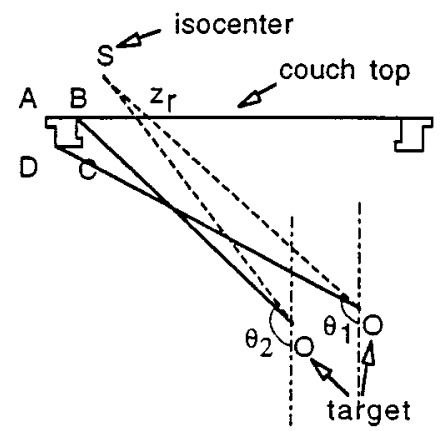

FIG. 2. (a) and (b): The geometry of couch-beam intersection gantry angle ranges. The target and the isocenter are located at $O$ and $S$, respectively. The variable $l_{a t}$ represents the lateral couch shift. The beam ray and other information displayed in these figures are the projections in the $x z$ plane at the isocenter (text).

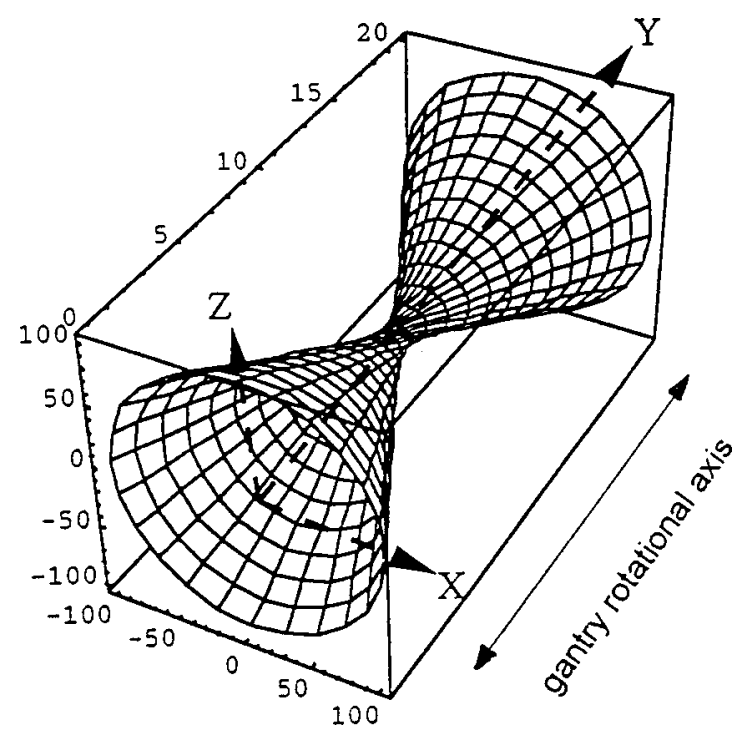

Fig. 3. The surface of revolution generated by a beam ray as a function of gantry rotation. The beam ray passes through the field point $\left(x_{g}^{\prime}, y_{g}^{\prime}, z_{g}^{\prime}\right)$ $=(10,10,0)$ in the gantry coordinate system. The coordinates of any point on the beam ray in the patient/fixed coordinate system are given by

$$
\left(\frac{x_{g}^{\prime}\left(S_{\mathrm{ad}}-z\right) \cos \theta}{S_{\mathrm{ad}}}+z \sin \theta, \frac{y_{g}^{\prime}\left(S_{\mathrm{ad}}-z\right)}{S_{\mathrm{ad}}}, \frac{x_{g}^{\prime}\left(z-S_{\mathrm{ad}}\right) \sin \theta}{S_{\mathrm{ad}}}+z \cos \theta\right)
$$

for the gantry rotation angle $\theta$. The $z$ coordinate of the beam ray shown varies from -100 to $100 \mathrm{~cm}$. The $y$ axis has been scaled by a factor of 2 compared to the $x$ and $z$ axes for clarity.

vertical distance between the corners $A$ and $D$ ) and couch half-width $\left(w_{d}\right.$, half the distance between the left corner $A$ and the right corner $A$ ), which are $2.7,10.0$, and $28.0 \mathrm{~cm}$, respectively, for the Varian Clinac 2100 C/D; In Figs. 2(a) and 2(b), the corner $C$ is shifted horizontally relative to the corner $A$ by $2 w_{b}$ and the corner $B$ is shifted horizontally from the corner $C$ by $1.2 \mathrm{~cm}$. Also the corner $E$ is shifted vertically up relative to $C$ by $1.0 \mathrm{~cm}$. Finally, the corner $D$ is shifted horizontally relative to corner $C$ by $1.9 \mathrm{~cm}$. The source to axis distance for the machine $\left(S_{\mathrm{ad}}\right)$ is $100 \mathrm{~cm}$. In addition, the model requires the input of the following variables: $\phi, \kappa$ : Couch and collimator rotation angles; $l_{a t}, z_{r}$ : Lateral translation of the couch and the height of the corner from the isocenter; and $X_{1}, X_{2}, Y_{1}, Y_{2}$ : The field size defined by the dual jaws at the isocenter. If the field size is to be defined through the MLC, individual leaf extensions are read from a file. All of the variables listed here take both positive and negative values except $S_{\text {ad }}$, which is a distance.

\section{A. Representation of a beam ray as a function of gantry rotation}

The first step in studying the problem of beam-PSA intersection is deriving and understanding the equation of a beam ray as a function of gantry rotation. A beam ray, being a straight line is defined by a point $\left(x_{c}, y_{c}, 0\right)$ at the boundary of the field edge in the collimator coordinate system. It is transformed in the gantry coordinate system as $\left(x_{g}^{\prime}, y_{g}^{\prime}, 0\right)$ $=\left(\left[x_{c} \cos \kappa-y_{c} \sin \kappa\right],\left[x_{c} \sin \kappa+y_{c} \cos \kappa\right], 0\right)$ for a collimator rotation angle of $\kappa$. This point when represented in the room coordinate system when the gantry is in a vertically irradiating position is 


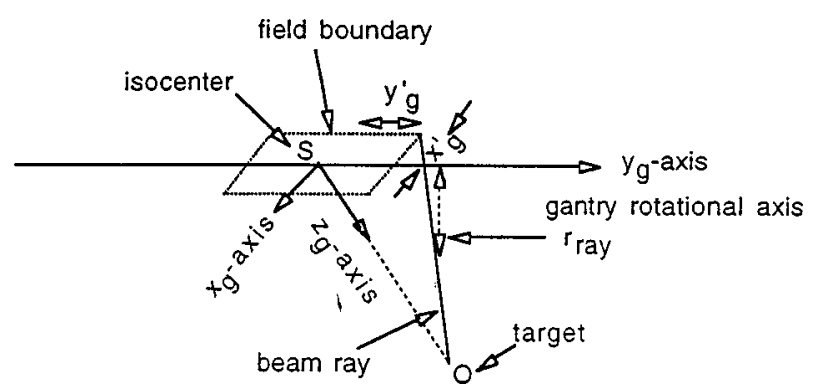

FIG. 4. An oblique view of the gantry system shown for a rectangular field. A beam ray is defined by a field point $\left(x_{g}^{\prime}, y_{g}^{\prime}, 0\right)$ in the plane of the isocenter. The $y_{g}$ axis here is defined to be along the rotational axis of the gantry through the isocenter. The distance $r_{\text {ray }}$ is the perpendicular distance from the $y_{g}$ axis to a point on the beam ray.

$$
\left(\frac{x_{g}^{\prime}\left(S_{\mathrm{ad}}-z\right)}{S_{\mathrm{ad}}}, \frac{y_{g}^{\prime}\left(S_{\mathrm{ad}}-z\right)}{S_{\mathrm{ad}}}, z\right) .
$$

The coordinates of this point in the beam ray is

$$
\begin{aligned}
& \left(\frac{x_{g}^{\prime}\left(S_{\mathrm{ad}}-z\right) \cos \theta}{S_{\mathrm{ad}}}\right. \\
& \left.\quad+z \sin \theta, \frac{y_{g}^{\prime}\left(S_{\mathrm{ad}}-z\right)}{S_{\mathrm{ad}}}, \frac{x_{g}^{\prime}\left(z-S_{\mathrm{ad}}\right) \sin \theta}{S_{\mathrm{ad}}}+z \cos \theta\right)
\end{aligned}
$$

for any gantry rotation angle $\theta$ in the room coordinate system. Each point in the beam ray defined by the above equation sweeps out a circle as the gantry rotates about the $y$ axis (Fig. 3). Since each point on the beam ray is at different radii from the $y$ axis, the beam ray sweeps out a surface revolution with a parabolic cross section (Fig. 3). This has the consequence that the intersection of the surface of revolution with the $x y$ plane of the room coordinate system is a parabola. In general, a horizontal line representing a couch edge intersects with the surface of revolution of the beam ray at two points. Therefore, there are two gantry angle solutions at which the beam ray intersects with the couch edge.

\section{B. Solution to the problem of beam ray intersection with a couch edge}

The gantry rotational axis, ( $y$ axis in Fig. 1) remains fixed in both room and gantry coordinate systems. In the gantry coordinate system, an equation for the perpendicular distance

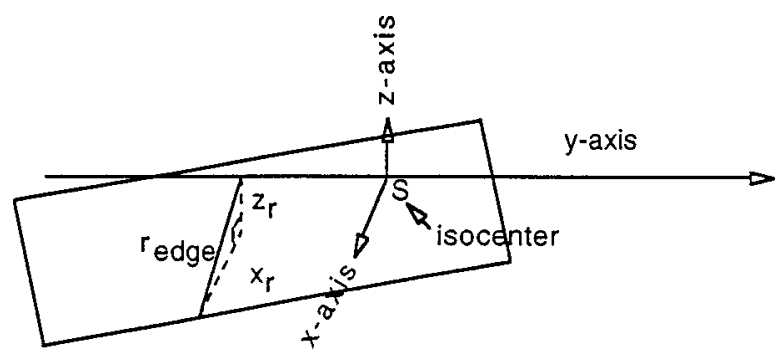

FIG. 5. The top view of couch system viewed directly above the isocenter. The couch is rotated through a rotation angle of $\phi$ degrees. $r_{\text {edge }}$ is the perpendicular distance from the $y$ axis to a point on the edge of the couch (where the beam ray intersects). The $x$ component of the intersection point is $x_{r}$. The vertical component of the couch location is $z_{r}$.

( $r_{\text {ray }}$ ) from the longitudinal axis to a point on the beam ray is derived here. In the room coordinate system an equation for the perpendicular distance ( $r_{\text {edge }}$ ) from the $y$ axis to a point on the couch edge is also derived. The condition of beamPSA (couch edge) intersection is given by $r_{\text {ray }}=r_{\text {edge }}$.

In Fig. 4, the gantry coordinate system (with the suffix $g$ ) defined at the isocenter rotates with the gantry (but not with the collimator). The equations of the beam ray in threedimensions in the gantry coordinate system are $z_{g}=S_{\mathrm{ad}}\left(y_{g}^{\prime}\right.$ $\left.-y_{g}\right) / y_{g}^{\prime}, y_{g}=y_{g}^{\prime} x_{g} / x_{g}^{\prime}$, and $x_{g}=x_{g}^{\prime} y_{g} / y_{g}^{\prime}$. The perpendicular distance from a point on the beam ray to the $y_{g}$ axis is given by $r_{\text {ray }}^{2}=z_{g}^{2}+x_{g}^{2}$. Upon substitution

$$
r_{\text {ray }}^{2}=S_{\mathrm{ad}}^{2}-\frac{2 S_{\mathrm{ad}}^{2} y_{g}}{y_{g}^{\prime}}+\frac{\left(x_{g}^{\prime 2}+S_{\mathrm{ad}}^{2}\right) y_{g}^{2}}{y_{g}^{\prime 2}} .
$$

In Fig. 5, the coordinate system shown is the room coordinate system defined at the isocenter. Let $r_{\text {edge }}$ be the perpendicular distance from a point on the edge of the couch to the $y$ axis. With the couch located at a vertical drop of $z_{r}$ from the isocenter, the equations of the edge in the room coordinate system are $x=a y+x_{\text {cou }}$ and $z=z_{r}$. Since $r_{\text {edge }}=z_{r}^{2}$ $+x^{2}$; upon substitution

$$
r_{\text {edge }}^{2}=z_{r}^{2}+x_{\text {cou }}^{2}+2 a y x_{\text {cou }}+a^{2} y^{2},
$$

where $a=\tan \phi$ and $x_{\text {cou }}=\left(w_{d}+l_{a t}\right) / \cos \phi$. The intersection of the surface of revolution of the ray (with the rotation of the gantry) and the couch edge is determined by $r_{\text {ray }}^{2}$ $=r_{\text {edge }}^{2}$. In the two coordinate systems in Figs. 4 and 5, the $y$ axes remain fixed, so $y=y_{g}$. From Eqs. (1) and (2), the intersection $\left(x_{r}, y_{r}\right)$ of the ray with the edge is $\left(y_{r}=y\right.$ $\left.=y_{g}\right)$

$$
y_{r}=y_{g}^{\prime} \frac{\left(a x_{\mathrm{cou}} y_{g}^{\prime}+S_{\mathrm{ad}}^{2}\right) \pm \sqrt{\left(a x_{\mathrm{cou}} y_{g}^{\prime}+S_{\mathrm{ad}}^{2}\right)^{2}-\left(x_{g}^{\prime 2}+S_{\mathrm{ad}}^{2}-a^{2} y_{g}^{\prime 2}\right)\left(S_{\mathrm{ad}}^{2}-z_{r}^{2}-x_{\mathrm{cou}}^{2}\right)}}{x_{g}^{\prime 2}+S_{\mathrm{ad}}^{2}-a^{2} y_{g}^{\prime 2}}
$$

and $x_{r}=a y_{r}+x_{\text {cou }}$.

There are two solutions to this quadratic equation. It is noted that even in the case of zero couch rotation $(a=0)$ there still are two solutions.

\section{Expression for gantry angle determination}

After the coordinates of the point of intersection of the ray and the edge have been determined, the corresponding gantry angle can be determined from the condition that the target, 
the point on the field edge and the point of intersection are in a straight line. The target and a field point which are $\left(0,0, S_{a d}\right)$ and $\left(x_{g}^{\prime}, y_{g}^{\prime}, 0\right)$, respectively, in the gantry coordinate system are converted to $\left(S_{\mathrm{ad}} \sin \theta, 0, S_{\mathrm{ad}} \cos \theta\right)$ and $\left(x_{g}^{\prime} \cos \theta, y_{g}^{\prime},-x_{g}^{\prime} \sin \theta\right)$ in the room coordinate system. The equations of the beam ray in three-dimension originating from the target and passing through the intersection point $\left(x_{r}, y_{r}, z_{r}\right)$ and the field point noted above are

$$
\begin{aligned}
\frac{y_{r}}{y_{g}^{\prime}} & =\frac{x_{r}-S_{\mathrm{ad}} \sin \theta}{x_{g}^{\prime} \cos \theta-S_{\mathrm{ad}} \sin \theta}, \\
\frac{y_{r}}{y_{g}^{\prime}} & =\frac{z_{r}-S_{\mathrm{ad}} \cos \theta}{-x_{g}^{\prime} \sin \theta-S_{\mathrm{ad}} \cos \theta} .
\end{aligned}
$$

By eliminating $y_{r}$, these two equations can be simplified to extract an expression for the intersecting gantry angle as

$$
\begin{aligned}
& \theta=-\alpha \pm \cos ^{-1}\left[\frac{S_{\mathrm{ad}} x_{g}^{\prime} \cos \alpha}{x_{g}^{\prime} z_{r}+x_{r} S_{\mathrm{ad}}}\right], \\
& \text { with } \alpha=\tan ^{-1}\left[\frac{z_{r} S_{\mathrm{ad}}-x_{g}^{\prime} x_{r}}{x_{g}^{\prime} z_{r}+x_{r} S_{\mathrm{ad}}}\right] .
\end{aligned}
$$

Out of the two possible solutions, the correct one is uniquely determined by the solution of the gantry angle that satisfies either Eq. (4) or Eq. (5). In this manner the two beam-PSA intersection solutions of a field point [Eq. (3)] lead to two unique gantry solutions. This is also true in the case of a field point that has the $y$ component $\left(y_{g}^{\prime}\right)$ zero. In this case there is only one point of beam-PSA intersection [Eq. (3)], but now both the solutions in Eq. (6) are valid.

\section{Consideration of any part of the field-PSA intersection}

The consideration of the problem of any part of a field intersecting with the couch edge is as follows. The entire field boundary is sampled at less than $3 \mathrm{~mm}$ intervals in the case of fields defined by jaws. For the field opening defined by the MLC leafs (of $1 \mathrm{~cm}$ field width at the isocenter for the Varian Clinac $2100 \mathrm{C} / \mathrm{D}$ ), the field boundary sampling is done at the center of an MLC leafs. The beam-PSA intersecting gantry angles using Eq. (6) depending upon the value of the variable $y_{r}$ (as explained in the previous section) are obtained. This is done for all of the field boundary points by incrementally moving through the MLC leaf boundary. In this manner a ranges of gantry angles of beam-couch edge intersection for variables $\theta$ are obtained. The conversion of the gantry angles to the readout convention of the Clinac $2100 \mathrm{C} / \mathrm{D}$ is performed by an addition of 360 degrees to the negative gantry angles.

\section{E. Consideration of the cross section of the PSA}

For the general cross-sectional shape of steel bars supporting the tennis racket, computing the beam intersection point can involve the following approach. This is to segment the steel bar cross section into increments and loop through

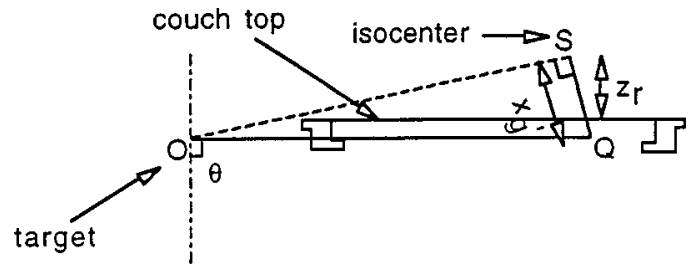

FIG. 6. This figure (projection in the $x z$ plane at the isocenter) shows the beam ray defined by the line $O Q$ that is parallel to the couch top. At lesser angle magnitudes, the beam ray would pass through the couch top before striking the PSA.

each point on the cross section for each of the elements at the field boundary to find the intersecting gantry angle range.

In the particular case of the Varian Clinac $2100 \mathrm{C} / \mathrm{D}$, with the corners $[A, B, C, D$, and $E$ in Figs. 2(a) and 2(b)] of the PSA so well defined, consideration of specific corners leads to the determination of beam-PSA intersecting gantry angle ranges. As the maximum lateral extension $(25 \mathrm{~cm})$ of the couch in $2100 \mathrm{C} / \mathrm{D}$ is less than the tennis racket half-width $(28 \mathrm{~cm})$, the isocenter is well inside of the corner $A$. These attributes of the couch make it simpler to determine what couch corners/edges are relevant for the determination of $\theta$. The consideration is to include an edge only if (a) the beam hits the edge before hitting the patient, i.e., enter through the bottom and exit through the top of the couch and (b) to select among the corners of PSA that lead to the largest gantry angle range. In this manner, to compute the beam-PSA intersecting most inclusive of the gantry angle range when the gantry head is on the right side in Figs. 2(a) and 2(b), beamPSA intersection with both right edge of the couch and the left edge were considered. For the right edge in Fig. 2(a), the corners $A, B, C$, and $E$ were considered and the most inclusive gantry angle range $\left(\theta_{1}, \theta_{2}\right)$ was generated. For the left edge in Fig. 2(b), the corners $B$ and $D$ of the left edge were considered and the most inclusive gantry angle range $\left(\theta_{1}, \theta_{2}\right)$ was generated. If there were an overlap between these two ranges then these two ranges were replaced by one gantry angle range inclusive of the two. For the right edge, corner $D$ was not considered as it is flanked on both sides by corners $A$ and $C$. The corners of the left edge other than $B$ and $D$ were not considered as the maximum range is defined by these two corners. Similar approach to solving beamPSA intersection was taken when the gantry head is located on the left side in Figs. 2(a) and 2(b).

In order for any part of the field edge to hit the PSA first before passing through the patient, the following constraint on the gantry angle is applied [Fig. 6 represents the situation described in Eq. (8)]

$$
\begin{aligned}
& \theta-\tan ^{-1}\left(\frac{x_{g}^{\prime}}{S_{\mathrm{ad}}}\right)>90^{\circ}, \quad \text { right steel bar, } \\
& \theta-\tan ^{-1}\left(\frac{x_{g}^{\prime}}{S_{\mathrm{ad}}}\right)<-90^{\circ}, \quad \text { left steel bar. }
\end{aligned}
$$


TABLE I. Measured and computed $x_{r}$ (see text) of the couch at the point of beam-PSA intersection and the intersecting gantry angle, $\theta_{1}$ are listed for various beam configurations involving the couch lateral displacement $\left(1_{a t}\right)$ of $-10 \mathrm{~cm}$. Angles and dimensions are represented in degrees and $\mathrm{cm}$, respectively.

\begin{tabular}{|c|c|c|c|c|c|c|}
\hline $\begin{array}{c}\text { Couch } \\
\text { height } \\
z_{r}\end{array}$ & $\begin{array}{c}\text { Couch } \\
\text { rot. } \\
\phi\end{array}$ & $\begin{array}{c}\text { Field } \\
\text { point } \\
\left(x_{g}^{\prime}, y_{g}^{\prime}\right)\end{array}$ & $\begin{array}{c}\text { Gantry } \\
\text { angle } \theta_{1} \\
\text { model }\end{array}$ & $\begin{array}{c}x_{r} \\
\text { model }\end{array}$ & $\begin{array}{c}\text { Gantry } \\
\text { angle } \theta_{1} \\
\text { meas. }\end{array}$ & $\begin{array}{c}x_{r} \\
\text { meas. }\end{array}$ \\
\hline-10 & -25 & $(-5,-5)$ & 105.6 & 21.6 & 105.7 & 21.8 \\
\hline-10 & -25 & $(-10,-10)$ & 95.8 & 23.3 & 96.1 & 23.5 \\
\hline-15 & 25 & $(5,-5)$ & 116.8 & 21.5 & 116.7 & 21.7 \\
\hline-15 & 25 & $(10,-10)$ & 107.5 & 23.2 & 107.4 & 23.4 \\
\hline
\end{tabular}

\section{RESULTS}

To verify that these two solutions do exist [Eq. (3)], measurements were performed for the setup: $z_{r}=-5 \mathrm{~cm}, l_{\text {at }}$ $=0 \mathrm{~cm}, \phi=10$ degrees and for a $10 \times 10 \mathrm{~cm}$ field size. The model predictions of $y$ were 3.5 and $6.4 \mathrm{~cm}$ for the field corner $(5,5,0)$. The corresponding gantry angles from Eq. (6) were 92.9 and 292.1 degrees. This agreed well with the measured values of $y$ of 3.5 and $6.3 \mathrm{~cm}$ and gantry angles of 93.2 and 292.9 degrees, respectively. The latter angle is treatable [Eq. (8)], because in this case the beam enters through the top of the couch before intersecting with the PSA. For the same setup with $z_{r}$ changed to $5 \mathrm{~cm}$, the model prediction of the gantry angle for the beam-PSA intersection point of $y=3.5 \mathrm{~cm}$, was 73.3 degrees and was in agreement with the measured value of 73.5 degrees. This example shows that the new model also works with the couch located above the isocenter. Since most clinical situations correspond to couch locations below the isocenter, such situations are more extensively studied in the next two paragraphs.

In Table I, measured and computed [from Eq. (3)] $x_{r}$ (the perpendicular distance from the $y z$ plane to the point of beam-PSA intersection) and the gantry angle $(\theta)$ are listed for various beam configurations involving the couch rotation. The light field is taken to represent the radiation field. Within the experimental uncertainty of about $2-4 \mathrm{~mm}$, there is a good agreement between the measured and computed values of $x_{r}$.

For the clinically relevant setups considered here, in general there are two sets of gantry angle ranges $(0<\theta \leqslant 180$ degrees and $180<\theta \leqslant 360$ degrees) corresponding to either side of the couch leading to beam-PSA intersection. Listed in Tables II and III are the model predictions [Eq. (6)] and measurements of a range for fields defined by dual independent jaws and an oval shaped MLC field, respectively. All dimensions are listed in $\mathrm{cm}$ and the angles are listed in degrees.

TABLE II. A comparison of model generated and measured range of gantry angles for which part of the beam passes through the couch support assembly for variety of situations. Angles and dimensions are represented in degrees and $\mathrm{cm}$, respectively.

\begin{tabular}{ccccccc}
\hline \hline $\begin{array}{c}\text { Couch } \\
\text { height } \\
z_{r}\end{array}$ & $\begin{array}{c}\text { Couch } \\
\text { lat. }\end{array}$ & $\begin{array}{c}\text { Couch } \\
\text { rot. } \\
l_{\text {at }}\end{array}$ & $\begin{array}{c}\text { Coll. } \\
\text { rot. } \\
\kappa\end{array}$ & $\begin{array}{c}\text { Jaws } \\
\left(X_{1}, X_{2}, Y_{1}, Y_{2}\right)\end{array}$ & $\begin{array}{c}\text { Gantry range } \\
\left(\theta_{1}, \theta_{2}\right) \\
\text { model }\end{array}$ & $\begin{array}{c}\text { Gantry range } \\
\left(\theta_{1}, \theta_{2}\right) \\
\text { meas. }\end{array}$ \\
\hline-10 & 0 & 0 & 0 & $(10,10,10,10)$ & $(264.2,214.8)$ & $(264.0,214.2)$ \\
-10 & 0 & 0 & 45 & $(10,10,10,10)$ & $(95.8,151.3)$ & $(96.3,151.0)$ \\
-10 & 10 & 0 & 0 & $(10,10,10,10)$ & $(264.1,192.7)$ & $(264.0,191.9)$ \\
-15 & 0 & 0 & 0 & $(10,10,10,10)$ & $(254.3,210.4)$ & $(254.0,210.0)$ \\
-10 & 0 & 0 & 0 & $(5,5,5,5)$ & $(257.1,221.7)$ & $(256.4,220.9)$ \\
-10 & 0 & 0 & 45 & $(5,10,5,10)$ & $(264.3,213.9)$ & $(263.7,213.6)$ \\
-10 & -10 & 25 & 0 & $(10,10,10,10)$ & $(95.8,173.9)$ & $(96.0,173.9)$ \\
-10 & -10 & 0 & 0 & $(10,10,10,10)$ & $(95.9,167.3)$ & $(95.6,167.1)$ \\
-10 & 5 & 10 & 0 & $(10,10,10,10)$ & $(264.2,202.5)$ & $(263.7,201.8)$ \\
-10 & 5 & 0 & 0 & $(10,10,10,10)$ & $(264.2,204.9)$ & $(263.6,203.9)$ \\
-10 & 0 & 10 & 20 & $(10,0,10,-5)$ & $(261.1,225.8)$ & $(260.8,225.4)$ \\
-10 & 0 & 0 & 20 & $(10,0,10,-5)$ & $(260.9,223.8)$ & $(260.3,223.4)$ \\
\hline \hline
\end{tabular}


TABLE III. A comparison of model generated and measured range of gantry angles for which part of the beam passes through the couch support assembly for various couch and collimator settings for an oval shaped MLC field. Angles and dimensions are represented in degrees and $\mathrm{cm}$, respectively.

\begin{tabular}{|c|c|c|c|c|c|}
\hline $\begin{array}{c}\text { Couch } \\
\text { height } \\
z_{r}\end{array}$ & $\begin{array}{c}\text { Couch } \\
\text { lat. } \\
l_{\text {at }}\end{array}$ & $\begin{array}{c}\text { Couch } \\
\text { rot. } \\
\phi\end{array}$ & $\begin{array}{c}\text { Coll. } \\
\text { rot. } \\
\kappa\end{array}$ & $\begin{array}{l}\text { Gantry range } \\
\left(\theta_{1}, \theta_{2}\right) \\
\text { model }\end{array}$ & $\begin{array}{c}\text { Gantry range } \\
\left(\theta_{1}, \theta_{2}\right) \\
\text { meas. }\end{array}$ \\
\hline-10 & 0 & 0 & 0 & $(100.1,141.1)$ & $(100.4,140.6)$ \\
\hline-10 & 0 & 0 & 45 & $(257.1,220.8)$ & $(256.7,219.8)$ \\
\hline-15 & 0 & 0 & 0 & $(109.5,146.0)$ & $(109.6,145.7)$ \\
\hline-10 & -10 & 0 & 0 & $(103.3,161.2)$ & $(103.4,160.9)$ \\
\hline-10 & -10 & 25 & 0 & $(102.5,158.6)$ & $(102.7,158.6)$ \\
\hline-10 & 0 & 0 & 320 & $(258.4,221.3)$ & $(257.7,220.3)$ \\
\hline-10 & 5 & 10 & 310 & $(255.7,214.5)$ & $(255.1,213.6)$ \\
\hline-10 & 5 & 0 & 310 & $(255.7,213.9)$ & $(255.1,212.8)$ \\
\hline-10 & -10 & 0 & 90 & $(107.9,155.5)$ & $(108.3,155.3)$ \\
\hline
\end{tabular}

\section{DISCUSSION}

Two gantry angle solutions for any given field point are possible even when the couch rotation angle is zero ( $a$ $=\tan \phi=0)$. This was not considered in the previous work. ${ }^{4}$

Normally, beam-PSA intersecting gantry angle range is determined by corners $A$ and $C$. However, there are also situations where one of the gantry angles determining the beam-PSA intersection is determined by corners either $B$ or $E$ as opposed to $C$. For the situation with $z_{r}=-5 \mathrm{~cm}, l_{\text {at }}$ $=-17.5 \mathrm{~cm}$, zero collimator and couch rotation angles and the vertical field half-widths $\left(x_{g}^{\prime}\right)$ of 4 and $-7 \mathrm{~cm}$, beamPSA intersecting gantry angle $\theta_{2}$ for the corners $C, B$, and $E$ were $183.75,185.98$, and 184.33 degrees, respectively. The middle one was taken as the solution as it leads to the largest gantry angle range.

The authors found that for treatable configurations in a $2100 \mathrm{C} / \mathrm{D}$ and for locations of couch below the isocenter (Tables II and III) the intersecting gantry angle range obtained for situation depicted in Fig. 2(b) was inclusive of the gantry angle range for the situation of Fig. 2(a). This may not be the case for locations of couch above the isocenter. Extended isocentric treatments ${ }^{1}$ can lead to setups where the couch top is located above the isocenter.

In addition to the measurement uncertainties of the machine parameters used in the model, the computational uncertainty in the model predictions also arise from finite sampling of the field size. For sampling distances of $\sim 3 \mathrm{~mm}$, the computational uncertainty in the model predictions of the gantry angles is about \pm 0.2 degrees. This was determined by reducing the sampling size and computing the intersecting gantry angle. There are two contributions to the uncertainties in the measured $x_{r}$ and the gantry angle, (1) the measurement uncertainties and (2) the setup uncertainties. There is about a $\pm 2 \mathrm{~mm}$ uncertainty in the measurement of $x_{r}$. The gantry angle at which the beam-PSA intersection occurs was observed from the light field edge. An uncertainty of about \pm 0.3 degrees was experimentally determined in this process. The other contribution to the uncertainty in the measurement process comes from accuracy of the gantry angles, which from the Varian $2100 \mathrm{C} / \mathrm{D}$ manual was \pm 0.5 degrees. From Eq. (6), it is seen that the uncertainties in $z_{r}, l_{\mathrm{at}}, S_{\mathrm{ad}}, x_{g}^{\prime}$, $y_{g}^{\prime}, \kappa$, and $\phi$ contribute to the setup uncertainties. According to the Varian $2100 \mathrm{C} / \mathrm{D}$ manual, $z_{r}, l_{\mathrm{at}}, S_{\mathrm{ad}}$ are specified within $\pm 2 \mathrm{~mm} ; X_{1}, Y_{1}, X_{2}, Y_{2}$ within $\pm 1 \mathrm{~mm}$ and the angles $\kappa$ and $\phi$ within \pm 0.5 degrees. The tennis racket is loosely mounted on the couch top. There is an uncertainty of about 1 $\mathrm{mm}$ in localizing it laterally.

Based on the above information and using Eq. (3), $x_{r}$ is determined within $\pm(2$ to 3$) \mathrm{mm}$ for couch rotation angles around zero and within $\pm(3$ to 4$) \mathrm{mm}$ for couch rotation angles around 25 degrees. So there is a good agreement between model predictions and data of $x_{r}$ in Table I. The uncertainty in the gantry angle [Eq. (6)] arising from setup and measurement uncertainties can be as much as 0.7 degrees. In the worst case scenario with all of them including the measurement uncertainty acting coherently and including the computational uncertainty in the model, the net uncertainty can be as much as \pm 1.7 degrees when jaws are used to define the field size. The model and data in Table II agree within \pm 1.0 degrees. When the MLC is used to define the field size, the field boundary sampling is done more coarsely and the net uncertainty in the gantry angle estimation can be greater than \pm 1.7 degrees. The model and the data in Table III agree within \pm 1.3 degrees.

The net uncertainty is smaller when comparing modelgenerated gantry angle range $\left(\theta_{1}, \theta_{2}\right)$ with the corresponding measured gantry angle range. This is because the settings of variables $l_{\text {at }}$ and $\phi$ are the same in the measurement of both $\theta_{1}$ and $\theta_{2}$. In the worst case scenario the measurement uncertainty arising from the light field matching is 0.6 degrees. The other setup and measurement uncertainty (from the gantry angle) can possibly increase this to at least 1.0 degree through coherent contributions. The model generated ranges and the data in Tables II and III are within \pm 0.8 degrees.

The importance of including the couch rotation in computing the range of gantry angles for which part of the beam passes through the PSA can be seen comparing rows 7 and 8 in Table II. Treatable configurations of no couch rotation and for a couch rotation angle of 25 degrees for a $20 \times 20 \mathrm{~cm}$ field size are compared here. The range of gantry angle is different by 6.7 degrees when the couch rotation is taken into account. Installation of accessory mounts for block trays or 
wedge significantly reduces the distance from the isocenter to the collimator head. This decreases the range of couch angles that are treatable when the gantry is located adjacent to the couch. This tends to reduce the difference in the range of gantry angles for couch with finite rotation and no rotation. The last two rows in Table II attest to the ability of the model to predict the intersecting gantry angles for asymmetric fields defined by the dual independent jaws.

The purpose of this work is to determine the beam-PSA intersection possibility for a certain gantry and couch configurations at the time of treatment planning. The chosen beams have to be first tested to see whether the patient can be setup for the treatment delivery. At the University of Pittsburgh an algorithm ${ }^{3}$ has been developed based on the work of Humm ${ }^{1}$ to determine the treatability of a beam configuration at the time of treatment planning. At the University of Michigan, Ann Arbor, a graphics simulation program ${ }^{2}$ has been developed and implemented to determine the treatability of a beam configuration. For couch angles much greater than 25 degrees the treatable gantry angles are such that the gantry is either directly below or above the couch. In that case there is very little possibility of beam-PSA intersection. If the beam is found to intersect the couch for a certain beam setup, the possibility of moving the couch a few $\mathrm{cm}$ laterally could be pursued. This changes $x_{r}$ and the gantry angle range of beam-PSA intersection and may make the beam setup treatable.

\section{ACKNOWLEDGMENTS}

The authors would like to thank Mary K. Martel for pointing out the existence of the previous work by Ellen Yorke. Also thanked are Benedick A. Fraass and Marc L. Kessler for reading the manuscript and providing useful comments. Supported in part by NIH Grant No. P01-CA59827.

\footnotetext{
a) Address for correspondence: Department of Radiation Oncology, 1 Gustave L. Levy Place, New York, NY 10029; electronic mail: moorthy_muthuswamy@smtplink.mssm.edu

${ }^{1}$ J. L. Humm, "Collision avoidance in computerized treatment planning," Med. Phys. 21, 1053-1064 (1994).

${ }^{2}$ M. L. Kessler, D. L. McShan, and B. A. Fraass, “A computer controlled conformal radiotherapy system. III: Graphical simulation and monitoring of treatment delivery,'’ Int. J. Radiat. Oncol., Biol., Phys. 33, 1173-1180 (1995).

${ }^{3}$ J. Bayouth, M. Muthuswamy, A. Kalend, M. Izadbakhsh, and J. Greenberger, "Computer controlled conformal therapy-Analytical and computer simulation of collision avoidance and treatment delivery,", Int. J. Radiat. Oncol., Biol., Phys. 32(S1), 188 (1995).

${ }^{4}$ E. D. Yorke, "The geometry of avoiding beam intersections and blocking tray collisions,'” Med. Phys. 16, 288-291 (1989).

${ }^{5} \mathrm{R}$. Siddon, "Solution to treatment planning problems using coordinate transformations,', Med. Phys. 8, 766-774 (1981).
} 